\title{
Review: interventions that lower cholesterol concentrations or blood pressure in diabetic patients prevent cardiovascular disease
}

Huang ES, Meigs JB, Singer DE. The effect of interventions to prevent cardiovascular disease in patients with type 2 diabetes mellitus. Am J Med 2001 Dec 1;111:633-42.

\section{QUESTION: In patients with type 2 diabetes mellitus, do medications for intensive reduction of cholesterol and glucose concentrations and blood pressure prevent cardiovascular disease (CVD)?}

\section{Data sources}

Studies were identified by searching Medline (1966 to 2000) and bibliographies of relevant studies and reviews.

\section{Study selection}

2 reviewers independently selected English-language randomised controlled trials (RCTs) that compared medications for intensive risk factor reduction with placebo or routine risk factor reduction in adults with type 2 diabetes; had $\geqslant 10$ patients in each group; followed patients for $\geqslant 1$ year; and reported on $\geqslant 1$ of aggregate cardiac events, cardiovascular mortality, all cause mortality, myocardial infarction, and stroke. Exclusion criteria were non-pharmacological treatments, absence of prespecified outcomes, or comparing therapeutic agents rather than evaluating risk factor lowering.

\section{Data extraction}

Data were extracted on patient treatment, CVD history, diabetes duration, risk factor levels, and outcome events.

\section{Main results}

7 cholesterol lowering RCTs (2603 diabetic patients, mean age 58 y, 87\% men), 6 blood pressure lowering RCTs (7572 diabetic patients, mean age 62 y, 57\% men), and 5 glucose lowering RCTs (5159 diabetic patients, mean age 57 y, $59 \%$ men) were included. Mean follow up ranged from 4.3 to 6.1 years for cholesterol lowering RCTs, 2.0 to 8.4 years for blood pressure lowering RCTs, and 1.0 to 12.5 years for glucose lowering RCTs. Pooled results showed that lowering cholesterol concentrations and blood pressure reduced cardiac event rates; the lowering of glucose concentrations did not reduce cardiac event rates (table). When cholesterol lowering RCTs were classified as primary prevention or secondary prevention, the effect remained for secondary prevention RCTs (3 RCTs) but not for primary prevention RCTs (2 RCTs) (table).

\section{Conclusions}

In patients with type 2 diabetes mellitus, medications to lower cholesterol concentrations or blood pressure substantially reduce cardiovascular disease. Medications to lower glucose concentrations appear to have less, if any, effect on cardiovascular disease.

\section{COMMENTARY} atherogenesis or vascular function, were not studied. concentrations remains unacceptably high.

1 Khaw KT, Wareham N, Luben R, et al. BMJ 2001;322:15-8.

3 Duckworth WC, McCarren M, Abraira C. Diabetes Care 2001;24:942-5.
Sources of funding: Public Health National Research Service Award;American Diabetes Association; SmithKline Beecham; Health Resources and Service Administration.

For correspondence: Dr E S Huang, University of Chicago, Chicago, Illinois, USA ehuang@medicine. bsd.uchicago.edu.

Despite a well known increase in the incidence of CVD in patients with type 2 diabetes, the relative effect of glycaemic control, cholesterol lowering, and blood pressure control with medications remains controversial. In their meta-analysis, Huang $e t$ al show that while cholesterol lowering and blood pressure lowering drugs reduced the risk for cardiac events, the risk reduction with glucose lowering agents was not statistically significant.

The interpretation of these findings is fraught with uncertainties in view of the following caveats. First, as pointed out by the authors, the underlying cardiovascular status of patients in each of the 3 interventions differed: the percentages of patients with coronary artery disease in the cholesterol lowering, blood pressure lowering, and glucose lowering RCTs were $89 \%, 32 \%$, and $13 \%$, respectively. Consequently, the lowest event rates in the control groups of the glucose lowering RCTs reflected the lowest absolute risk for events in patients with such underlying disease. Only 2 of the 5 glucose lowering RCTs, both targeted at primary prevention, provided aggregate cardiac end points. In fact, in the only secondary glucose lowering RCT included in this analysis, the reduction in cardiovascular mortality was statistically significant; however, it was a relatively short term RCT. Second, the mean fasting plasma glucose reduction of $29 \mathrm{mg} / \mathrm{dl}$ and mean glycated haemoglobin $\left(\mathrm{HbA}_{1}\right)$ reduction of $0.9 \%$ achieved in the glucose lowering trials may not sufficiently improve the glycaemic burden. Finally, the effects of newer insulin sensitising agents (eg, thiazolidinediones), with potential benefits for

Epidemiological evidence continues to show that the increased risk for CVD extends well into the moderately increased range of glycaemia $\left(\mathrm{HbA}_{1 \mathrm{c}}<7.0 \%\right)$. ${ }^{1}$ Thus far, none of the RCTs available have fully tested the glucose lowering hypothesis. Currently, 2 large trials aiming for tighter glucose control with various pharmacological agents, including insulin sensitisers, are being done..$^{3}$ Furthermore, despite evidence for reduced cardiovascular events with cholesterol lowering and blood pressure lowering medications, the residual risk in diabetic patients treated to current target

Om P Ganda, MD Joslin Diabetes Center, Boston, Massachusetts, USA

2 Action to Control Cardiovascular Risk in Diabetes (ACCORD). (www.accordtrial.org).

Intensive risk factor reduction (treatment) v placebo or routine risk factor reduction (control) for aggregate cardiac events* in type 2 diabetes at mean 1 to 12.5 years of follow upt

\begin{tabular}{lcccccc} 
& & \multicolumn{2}{c}{ Events/1000 person years } & & \\
\cline { 3 - 6 } Risk factor interventions & Number of studies & Treatment & Control & Summary rate ratio (95\% Cl) & Person-years needed to treat (CI) \\
Cholesterol lowering & 5 & 30 & 41 & 0.75 (0.61 to 0.93) & 106 (62 to 366$)$ \\
\hline Primary prevention & 2 & 8 & 19 & $0.44(0.17$ to 1.2$)$ & 97 (45 to NM) \\
\hline Secondary prevention & 3 & 34 & 44 & $0.77(0.62$ to 0.96$)$ & 120 (61 to 48056$)$ \\
\hline Blood pressure lowering & 3 & 17 & 23 & $0.73(0.57$ to 0.94$)$ & 157 (88 to 726$)$ \\
\hline Glucose lowering & 2 & 15 & 18 & $0.87(0.74$ to 1.01$)$ & Not significant \\
\hline
\end{tabular}

${ }^{*}$ Death from coronary heart disease and non-fatal myocardial infarction. $†$ Summary rate ratios were calculated using a fixed effects model. NM = not clinically meaningful because person years needed to treat is negative. 